\title{
Dubins Paths Through a Sequence of Points: Lower and Upper Bounds
}

\author{
Satyanarayana Manyam ${ }^{1}$
}

\author{
Sivakumar Rathinam ${ }^{2}$
}

\author{
David Casbeer $^{1}$
}

\begin{abstract}
This article addresses an important path planning problem for robots and Unmanned Aerial Vehicles (UAVs) which aims to find a shortest path of bounded curvature passing through a given sequence of target points on a ground plane. Currently, there is no algorithm that can compute an optimal solution to this problem. Therefore, tight lower bounds are vital in determining the quality of any feasible solution to this problem. Novel tight lower bounding algorithms are presented in this article by relaxing some of the heading angle constraints at the target points. The proposed approach requires us to solve variants of an optimization problem called the Dubins interval problem between two points where the heading angles at the points are constrained to be within a specified interval. These variants can be solved using tools from optimal control theory. Specifically, two lower bounding algorithms are presented in this article using this approach and these bounds are then compared with the existing results in the literature. Computational results are also presented to corroborate the performance of the proposed algorithms.
\end{abstract}

\section{INTRODUCTION}

This article addresses the problem of finding a path of minimum length for a vehicle (car like robot) that needs to visit a given sequence of points. The vehicle is modeled as a point robot, i.e., we ignore the dynamics of the vehicle and consider only its kinematic constraints. There are also no disturbances or obstacles in the environment. The path of the vehicle must satisfy a kinematic constraint which limits the turning radius of the vehicle at any point along its path. This problem belongs to a well known class of motion constrained UAV (Unmanned Aerial Vehicle) routing problems that have received significant attention in the literature over the last decade [1]-[9].

The problem of finding a shortest path for the vehicle to travel from a target at $\left(x_{1}, y_{1}\right)$ with heading angle $\theta_{1}$ to another target at $\left(x_{2}, y_{2}\right)$ with heading angle $\theta_{2}$ subject to the turning radius constraint of the vehicle has received significant attention in the literature over the last 50 years. L.E. Dubins [10] first solved this problem in 1957 and showed that an optimal path must consist of at most three segments and each of the segments must be either an arc of minimum turning radius (denoted as $C$ ) or a straight line (denoted as $S$ ). Specifically, Dubins [10] showed that the optimal path must either be of type $C S C$ or $C C C$, or a degenerate form of these paths. An optimal path of the type $C S C$ or $C C C$ is typically referred to as a Dubins path in the literature. An alternate proof of this result by Dubins was developed by Boissonnat et. al in [11] using optimal

\footnotetext{
${ }^{1}$ Control Science Center of Excellence, Air Force Research Laboratory, WPAFB,OH.

${ }^{2}$ Department of Mechanical Engineering, Texas A\&M University, College Station, TX.
}

control theory. There have also been extensions of these results for other vehicles; for example, in [12], Sussmann and Tang solved this shortest path problem for a vehicle that travels both forwards and backwards with the turning radius constraint. Variants of this problem in the presence of obstacles were also addressed in [13]-[15].

This article addresses an important generalization of the above problem where one aims to find a shortest path through the given sequence of target points subject to the turning radius constraint of the vehicle. We refer to this problem as the Generalized Dubins Path Problem (GDPP) in this article. This problem was first addressed by Lee et. al. in [16] where the authors present a 5.03-approximation algorithm. An $\alpha$ approximation algorithm for a minimization problem is an algorithm that runs in polynomial time and finds a feasible solution whose cost is at most $\alpha$ times the optimal cost for the problem. This algorithm by Lee et. al [16] remains the only constant factor algorithm in the literature for the GDPP to date. For the special case when the Euclidean distance between any two adjacent points in the given sequence is at least equal to four times the minimum turning radius, Goaoc et al. [17] have recently shown that the optimal cost of the GDPP is convex in the heading angles at each target and that a gradient descent algorithm can find an optimum.

A generalization of the GDPP where a set of points to be visited is given but the sequence is not given, and the vehicle has to return to its starting location after visiting all the points is the Dubins Traveling Salesman Problem (DTSP). DTSP has received considerable attention primarily due to its application in robotic and UAV path planning applications. Heuristics and approximation algorithms to solve the DTSP and its variants are presented in [8], [9], [18]-[21]; lower bounds to this problem are computed using Lagrangian relaxation in [22] and by relaxing the heading angle constraint at target points in [23].

Despite the numerous results available for finding feasible solutions for the GDPP (one can refer to [23] for a review of the methods available), there is currently no algorithm that can find an optimal solution to the GDPP. Therefore, it becomes critically important to develop algorithms or computationally efficient procedures that can provide tight lower bounds to the GDPP. This will help us to evaluate the quality of the feasible solutions with respect to the lower bounds.

A trivial lower bound can be obtained by simply removing the turning radius constraint of the vehicle; in this case, the lower bound is simply the sum of all the Euclidean distances of the adjacent points in the given sequence. Numerical results (you can refer to the results section of 
this article) show that this trivial bound is quite conservative, and therefore, serves as a poor estimate of the optimal cost. Recently, a novel procedure was presented for developing tight lower bounds for the DTSP [23] by relaxing some of the angle constraints at each point. In this article, we generalize this procedure and develop other lower bounds for the GDPP. The following are the contributions of this article:

- At each given point, the heading angle of the path that ends (incoming angle) at the point should be the same as the heading angle at the beginning of the path that starts (outgoing angle) at the point. We relax this constraint at some points in the given sequence by allowing the incoming and the outgoing angles to be different but restricting their absolute difference to be within a given interval. We then pose the resulting relaxed problem as a shortest path problem. As any optimal solution to the GDPP is also a feasible solution to this relaxed problem, one obtains a lower bound. This procedure generalizes the result in [23].

- All the bounds have been implemented to corroborate the performance of the proposed algorithms.

This article is organized as follows: the problem of computing lower bound is first presented in Section II. In Section III-B, we address a sub-problem that arises while computing the lower bound called the Dubins Interval Problem. The algorithms to compute lower bounds are presented in Section IV and the computational results are presented in Section VI. The conclusions are finally made in Section VII.

\section{Problem Formulation}

Let $T=\left\{t_{1}, t_{2}, \ldots, t_{n}\right\}$ be the given sequence of $n$ target points. An optimal path for the GDPP is a curve of shortest length and bounded curvature that passes through all of these target points. We aim to find a tight lower bound to the length of this optimal path by relaxing certain constraints along the path. This optimal path is a concatenation of $n-1$ individual Dubins paths $\left\{d_{12}, d_{23}, \ldots d_{(n-1) n}\right\}$, where $d_{i j}$ is a Dubins path from target $i$ to $j$. Let $\theta_{i}, i=1 \ldots n$, be the angle made by the tangent of the path at each point with respect to the global $x$-axis. If the values of $\theta_{i}$ 's are given, one can construct each individual Dubins paths using the result in [10], and hence compute an optimal path for the GDPP. Therefore, the variables to be determined to find an optimal path are the heading angles $\left(\theta_{i}\right.$ 's) at the target points.

Let targets $t_{i}, t_{j}, t_{k}$ be in a sequence, and let $\theta_{i}, \theta_{j}$ and $\theta_{k}$ be the headings of the path at these targets. Let $\theta_{j a}$ represents the final heading of the path $d_{i j}$, and $\theta_{j d}$ be the initial heading of the path $d_{j k}$. The path is feasible only if these two headings are equal, $\theta_{j a}=\theta_{j d}=\theta_{j}$. One can compute a lower bound to the optimal Dubins path by relaxing this constraint, i.e. at every intermediate point, the incoming angle can be different from the outgoing angle. This leads to a trivial lower bound, which is the sum of the Euclidean distances along the given sequence of points. We aim to find a tighter lower bound using the following relaxation: The incoming and outgoing headings may not be equal, but should be within a specified interval. A solution to the Dubins path problem with this relaxation is shown in Figure 1.

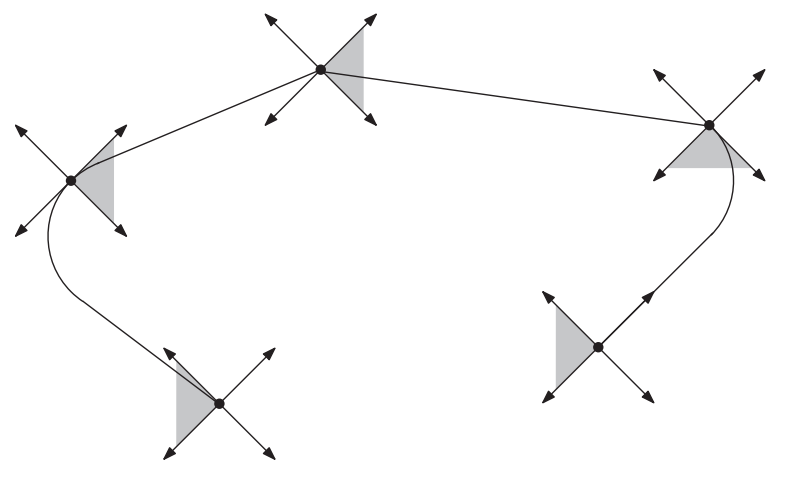

Fig. 1. Relaxed Dubins path

In general, tight lower bounds are found by relaxing the minimal number of constraints possible. We provide two algorithms to compute lower bounds, each with different set of relaxations. In the first algorithm, we relax the heading angle constraint at every point, and in the second we relax it only at alternate points. The set of heading angles $[0,2 \pi)$ at a target $t_{i}$ is partitioned into a set of intervals $\mathcal{I}_{i}=\left\{\left[0, \phi_{i 1}\right],\left[\phi_{i 1}, \phi_{i 2}\right], \ldots\left[\phi_{i m-1}, \phi_{i m}=2 \pi\right]\right\}$, such that $0 \leq \phi_{i 1} \leq \phi_{i 2} \ldots \phi_{i m}=2 \pi$. This partitioning is done at every point in the first relaxation criteria, and only at alternate points $t_{1}, t_{3}, \ldots$ in the second relaxation criteria. We formulate the optimization problems in each of these relaxations in the ensuing discussion:

\section{A. Relaxing the angles at every point}

Let $\left(x_{v}, y_{v}\right)$ be the $x y$-coordinates of target $t_{v}$ in the global reference frame, let $\theta_{v a}$ denote the arrival (incoming) heading angle at any target $t_{v}$, and $\theta_{v d}$ denote the departure (outgoing) heading angle. In the first relaxation criteria, for every pair of successive target points $\left(t_{i}, t_{j}\right)$ and a given interval of headings $I_{i}, I_{j}$ at those points, we need to solve the two point Dubins interval problem, i.e. find the shortest Dubins path $d_{i j}^{*}$ between two points where the headings at those points belong to the specified intervals.

$$
d_{i j}^{*}\left(I_{i}, I_{j}\right)=\min _{\theta_{i d} \in I_{i}, \theta_{j a} \in I_{j}} d_{i j}\left(\theta_{i d}, \theta_{j a}\right)
$$

where, $d_{i j}\left(\theta_{i d}, \theta_{j a}\right)$ is the Dubins path between $t_{i}$ and $t_{j}$ with headings $\theta_{i d}$ at $t_{i}$ and $\theta_{j a}$ at $t_{j}$. A lower bound to the GDPP is given by the minimum of the summation $\sum_{i=1 . . n-1} d_{i i+1}^{*}\left(I_{i}, I_{i+1}\right)$ over all possible intervals, $I_{i}$ at all points $i=1, \ldots, n$.

\section{B. Relaxing the angles at alternate points}

Suppose $t_{i}, t_{j}, t_{k}$ are in a sequence and the headings are partitioned at targets $t_{i}$ and $t_{k}$. Let $d_{i j k}\left(\theta_{i d}, \theta_{k a}\right)$ denote the Dubins path from $\left(x_{i}, y_{i}\right)$ to $\left(x_{k}, y_{k}\right)$ with heading angles $\theta_{i d}$ at $t_{i}$ and $\theta_{k a}$ at $t_{k}$, and it passes through the point $\left(x_{j}, y_{j}\right)$. We need to find the shortest Dubins path from $t_{i}$ to $t_{k}$, such 
that the angle $\theta_{i d}$ at $t_{i}$ and $\theta_{k a}$ at $t_{k}$ belongs to given intervals $I_{i}$ and $I_{k}$ respectively. Let us denote this as $d_{i j k}^{*}\left(I_{i}, I_{k}\right)$

$$
d_{i j k}^{*}\left(I_{i}, I_{k}\right):=\min _{\theta_{i d} \in I_{i}, \theta_{k a} \in I_{k}} d_{i j k}\left(\theta_{i d}, \theta_{k a}\right) .
$$

The problem stated in the above equation is referred to as the three point Dubins interval problem. The minimum of the summation of Dubins paths $\sum_{i=1,3 . . n-2} d_{i(i+1)(i+2)}^{*}\left(I_{i}, I_{i+2}\right)$ over all the intervals at alternate points, $I_{i}, I_{i+2}, \ldots$, gives another lower bound to the GDPP.

To compute the lower bounds, first we need to solve the two point Dubins interval problem and the three point Dubins interval problem.

\section{DUBINS INTERVAL PROBLEM}

\section{A. Two point Dubins interval problem}

The objective of the two point Dubins interval problem is to solve $\min _{\theta_{1} \in I_{1}, \theta_{2} \in I_{2}} d_{12}\left(\theta_{1}, \theta_{2}\right)$. Let the intervals be denoted as $I_{1}=\left[\theta_{1}^{\min }, \theta_{1}^{\max }\right]$ and $I_{2}=\left[\theta_{2}^{\min }, \theta_{2}^{\max }\right]$. Also, let $I_{1}^{o}=\left(\theta_{1}^{\min }, \theta_{1}^{\max }\right)$ and $I_{2}^{o}=\left(\theta_{2}^{\min }, \theta_{2}^{\max }\right)$. We recently solved this problem using geometrical analysis in [23] and using optimal control theory in [24]. Here, we present the main result in the following theorem:

Theorem 3.1: Suppose $L$ and $R$ represents the left (counter-clockwise) and the right (clockwise) circular arcs with radius equal to the minimum turning radius of the vehicle, and $S$ represents a straight line. More specifically, $L_{\psi}$ and $R_{\psi}$ are the arcs subtended by angle $\psi$. The shortest path of bounded curvature between the two targets with the departure angle $\theta_{1} \in \Theta_{1}=\left[\theta_{1}^{\min }, \theta_{1}^{\max }\right]$ at target 1 and the arrival angle $\theta_{2} \in \Theta_{2}=\left[\theta_{2}^{\min }, \theta_{2}^{\max }\right]$ at target 2 must be one of the following candidate solutions or a degenerate form of these:

Case 1: $S$ or $L_{\psi}$ or $R_{\psi}$ or $L_{\psi} R_{\psi}$ or $R_{\psi} L_{\psi}$ with $\psi>\pi$.

Case 2: $\theta_{1}=\theta_{1}^{\max }$ and $\theta_{2}=\theta_{2}^{\max }$ and the path is $L S R$.

Case 3: $\theta_{1}=\theta_{1}^{\max }$ and $\theta_{2}=\theta_{2}^{\min }$ and the path is either $L S L$ or $L R_{\psi} L$ with $\psi>\pi$.

Case 4: $\theta_{1}=\theta_{1}^{m i n}$ and $\theta_{2}=\theta_{2}^{\min }$ and the path is $R S L$.

Case 5: $\theta_{1}=\theta_{1}^{\min }$ and $\theta_{2}=\theta_{2}^{\max }$ and the path is either $R S R$ or $R L_{\psi} R$ with $\psi>\pi$.

Case 6: $\theta_{1}=\theta_{1}^{\max }$ and $\theta_{2} \in I_{2}^{o}$ and the path is either $L S$ or $L R_{\psi}$ with $\psi>\pi$.

Case 7: $\theta_{1}=\theta_{1}^{\text {min }}$ and $\theta_{2} \in I_{2}^{o}$ and the path is either $R S$ or $R L_{\psi}$ with $\psi>\pi$.

Case 8: $\theta_{1} \in I_{1}^{o}$ and $\theta_{2}=\theta_{2}^{\max }$ and the path is either $S R$ or $L_{\psi} R$ with $\psi>\pi$.

Case 9: $\theta_{1} \in I_{1}^{o}$ and $\theta_{2}=\theta_{2}^{\min }$ and the path is either $S L$ or $R_{\psi} L$ with $\psi>\pi$.

\section{B. Three point Dubins interval problem}

We will state the three point Dubins interval problem as the following: given the locations of three points $\left(t_{i}, t_{j}, t_{k}\right)$, and two intervals $I_{i}, I_{k} \subset[0,2 \pi]$ at $t_{i}$ and $t_{k}$ respectively, find the shortest path such that $(i)$ its end points are $t_{i}$ and $t_{k}$, and it passes through the point $t_{j}$, such that $\theta_{j a}=\theta_{j d},(i i)$ its heading angles at initial and final points should lie within the given intervals $\theta_{i} \in I_{i}, \theta_{k} \in I_{k}$, (iii) the radius of curvature at any point along the path is at least greater than a specified constant. Here, the intervals $I_{i}$ and $I_{k}$ are continuous and can be specified as $\left[\theta_{i}^{\min }, \theta_{i}^{\max }\right]$ and $\left[\theta_{k}^{\min }, \theta_{k}^{\max }\right]$ respectively. A sample solution to the three point Dubins interval problem is shown in Figure 2.

The variables to be determined in this problem are the heading angles at the three points $\theta_{i}, \theta_{j}$ and $\theta_{k}$. If they are known, we can construct the shortest path using the Dubins result. We refer to the location of the points and the tangent angle together as a configuration $(x, y, \theta)$. We

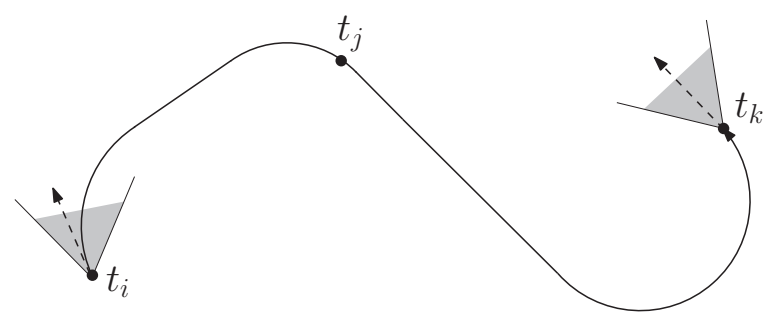

Fig. 2. Three point interval to interval Dubins path

aim to solve this by breaking the path into two parts, the path from $t_{i}$ to $t_{j}$ and $t_{j}$ to $t_{k}$. We discretize the angle at the intermediate point $\theta_{j}$, i.e., pick a discrete set of angles $\Psi=\left\{\psi_{1}, \ldots \psi_{p}\right\}, \psi_{i} \in[0,2 \pi]$. For each angle $\psi_{j} \in \Psi$, we compute two paths $(i)$ shortest path from $t_{i}$ to $t_{j}$, with initial heading angle $\theta_{i} \in I_{i}$ i.e $\bar{d}_{i j}\left(I_{i}, \psi_{j}\right)=$ $\min _{\theta_{i} \in I_{i}} d_{i j}\left(\theta_{i}, \psi_{j}\right)$, and (ii) path from $t_{j}$ to $t_{k}$ with final heading angle $\theta_{k} \in I_{k}, \bar{d}_{j k}\left(\psi_{j}, I_{k}\right)=\min _{\theta_{k} \in I_{k}} d_{j k}\left(\psi_{j}, \theta_{k}\right)$. We pick the heading angle at intermediate point, such that the sum of the length of the two paths is minimum, $d_{i j k}^{*}\left(I_{i}, I_{k}\right):=\min _{\psi_{j} \in \Psi}\left[\bar{d}_{i j}\left(I_{i}, \psi_{j}\right)+\bar{d}_{j k}\left(\psi_{j}, I_{k}\right)\right]$.

Here, there are two sub-problems and we have to find the shortest Dubins paths in each. In the first sub-problem, the heading angle at the initial location is restricted to an interval of angles and the heading at the final location is given; where as in the second sub-problem, the heading at initial point is given and the heading at final point is restricted to an interval. Though they seem to be different problems, for a generic case considered herein, the optimal solution of one of them corresponds to the optimal solution of the other.

Let $t_{i}$ and $t_{j}$ be two points with coordinates $\left(x_{i}, y_{i}\right)$ and $\left(x_{j}, y_{j}\right)$ respectively. Let $\theta_{j}$ be the given heading angle at $t_{j}$ and $\left[\theta_{i}^{\min }, \theta_{i}^{\max }\right]$ be the interval of headings given at $t_{i}$.

Proposition 1: The shortest Dubins path from $t_{i}$ to $t_{j}$ with a heading $\theta_{i} \in\left[\theta_{i}^{\min }, \theta_{i}^{\max }\right]$ at $t_{i}$ and $\theta_{j}$ at $t_{j}$, and the shortest path from $t_{j}$ to $t_{i}$ with heading $\theta_{j}+\pi$ at $t_{j}$ and $\theta_{i}+\pi \in\left[\theta_{i}^{\min }+\pi, \theta_{i}^{\max }+\pi\right]$ at $t_{i}$ are isometric. 


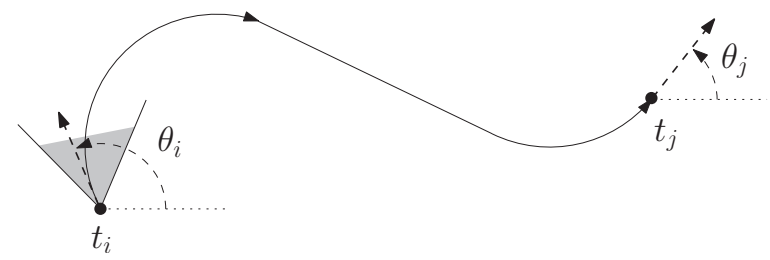

(a) Dubins path with heading at the initial point restricted to an interval and heading at final point given

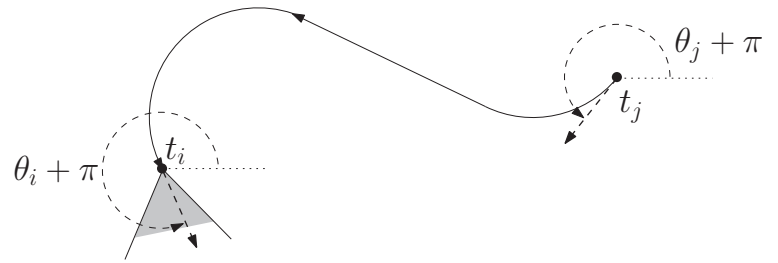

(b) Dubins path with heading at the initial point given and heading at final point restricted to an interval

Fig. 3. Equivalent Dubins paths between two points

Proof: From symmetry and Figure. 3 those two paths are equivalent.

We will attempt to solve the subproblem to find shortest Dubins path with a specified heading at the initial point and the heading at final point is constrained to be in an interval. In the following section, we formally state the problem.

1) Configuration-to-interval Dubins path problem: The paths with bounded curvature can be modeled using a set of kinematic constraints. Here, we assume that the vehicle travels at constant speed and therefore the length of the path is a constant times the time of travel. Let $(x(t), y(t), \theta(t))$ be the states of the vehicle, where $(x(t), y(t))$ are the coordinates of the vehicle in the global frame, and $\theta(t)$ is the heading angle with respect to the $x$-axis. Let $v_{0}$ be the speed of the vehicle and the shortest path problem is posed as an optimal control problem as shown:

$$
\min _{u(t) \in\left[-\frac{v_{o}}{\rho}, \frac{v_{o}}{\rho}\right]} \int_{0}^{T} 1 d t
$$

subject to

$$
\begin{aligned}
& \dot{x}=v_{o} \cos \theta, \\
& \dot{y}=v_{o} \sin \theta, \\
& \dot{\theta}=u, \\
& x(0)=x_{1}, \quad x(T)=x_{2}, \\
& y(0)=y_{1}, \quad y(T)=y_{2}, \\
& \theta(0)=\theta_{1}, \\
& \theta_{2}^{\text {min }}-\theta(T) \leq 0, \\
& \theta(T)-\theta_{2}^{\text {max }} \leq 0 .
\end{aligned}
$$

Here, the rate of change of heading $(u(t))$ is the control input and it is bounded $u(t) \in\left[-\frac{v_{o}}{\rho}, \frac{v_{o}}{\rho}\right]$, and $\rho$ is the minimum turning radius of the vehicle. This problem is a special case of the Dubins interval problem [24], where in the locations of the initial and final point are given and the headings at both of the points are restricted to two specified intervals. Here, we need to solve a special case of the Dubins interval problem where the heading angle at the initial point is given. We state the theorem from [24] that characterizes the solution of this special case:

Theorem 3.2: Any shortest path which is $C^{1}$ and piecewise $C^{2}$ of bounded curvature between two points with the departure angle $\theta_{1}$ at point $t_{1}$ and the arrival angle $\theta_{2} \in \Theta_{2}=\left[\theta_{2}^{\min }, \theta_{2}^{\max }\right]$ at point $t_{2}$ must be one of the following or a degenerate form of these:

Case 1: The path is either $L S$ or $R S$ or $L R_{\psi}$ or $R L_{\psi}$ with $\psi>\pi$.

Case 2: $\theta_{2}=\theta_{2}^{\max }$ and the path is either $L S R$ or $R S R$ or $R L_{\psi} R$ with $\psi>\pi$.

Case 3: $\theta_{2}=\theta_{2}^{\min }$ and the path is either $R S L$ or $L S L$ or $L R_{\psi} L$ with $\psi>\pi$.

\section{COMPUTATION OF LOWER BOUNDS FROM THE RELAXATIONS}

To implement the relaxations, we divide the possible headings $[0,2 \pi]$ at target points into a set of $p$ intervals; this is done at every point in the first relaxation, referred to as 2-point relax, and at alternate points in the second relaxation, referred to as 3-point relax. We chose the intervals at each point to be of same size (uniform intervals), and the same intervals are chosen at all the relaxed points. At each relaxed point $t_{i}$, the set of intervals would be $\mathcal{I}_{i}=\{[0=$ $\left.\left.\phi_{i 0}, \phi_{i 1}\right],\left[\phi_{i 1}, \phi_{i 2}\right], \ldots\left[\phi_{i(p-1)}, \phi_{i p}=2 \pi\right]\right\}$. The size of the intervals is $\phi_{i 1}-\phi_{i 0}=\phi_{i 2}-\phi_{i 1}=\ldots \phi_{i p}-\phi_{i(p-1)}=$ $\phi_{d}$ (constant), $\forall i=1 \ldots n$. Now, we compute the lower bound to the Dubins path problem by constructing a graph $G$, and solving a corresponding shortest path problem on $G$. The algorithms Relax-2-point and Relax-3-point present the construction of graphs based on the two different relaxations, and a lower bound to the GDPP is computed by finding the length of the shortest path on these graphs.

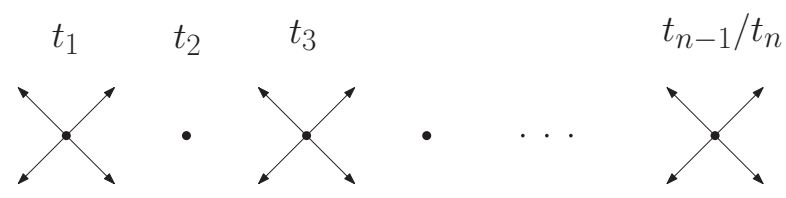

(a)
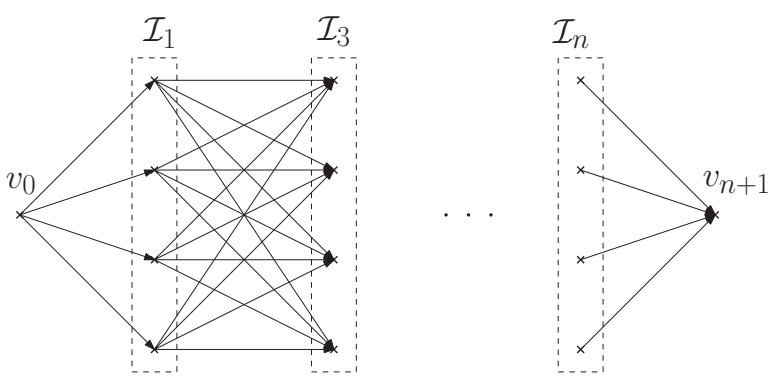

(b)

Fig. 4. Graph construction to compute lower bound 


\section{Algorithm: Relax-2-point}

1) Divide the set of heading angles at each point $t_{i}$ and create the set of intervals $\mathcal{I}_{i}=\left\{I_{i 1}, \ldots I_{i p}\right\}$ for $i=1 \ldots n$.

2) Construct a graph $G^{1}$ as the following: add a node to $G^{1}$ corresponding to each interval $I_{i k} \in \mathcal{I}_{i}, \forall i=$ $1 \ldots n, k=1 \ldots p$; add an edge between two nodes corresponding to any two interval $I_{i m}, I_{i+1 n}$, if they belong to interval sets $\mathcal{I}_{i}$ and $\mathcal{I}_{i+1}$ at successive points; set the weight of the edge to be the distance of the shortest Dubins path between the interval $I_{i m}$ to $I_{i+1 n}$, which is computed using the result in [24].

3 ) Add an auxiliary node $v_{0}$, and add edges from $v_{0}$ to every node corresponding to all of the intervals in the set $\mathcal{I}_{1}$. Set the weight of all these edges to be zero.

4) Add an auxiliary node $v_{n+1}$, and add edges from all the nodes corresponding to the intervals in $\mathcal{I}_{n}$ to $v_{n+1}$; set the weight of these edges to be zero.

5) Compute the shortest path on $G^{1}$ from $v_{0}$ to $v_{n+1}$ using the Dijkstra's algorithm.

\section{COMPUTATION OF A LOWER BOUND USING THE APPROXIMATION ALgORITHM IN [16]}

In this section, we briefly outline a lower bound we compute from the approximation algorithm presented in [16]. The algorithm in [16] first identifies all the subsequences of points where the distance between any two adjacent points in each subsequence is at most equal to twice the minimum radius of the vehicle. Given such a subsequence, the algorithm further decomposes this subsequence into smaller subsequences, say $S_{1}, S_{2}, \cdots, S_{r}$ where one of the following properties is guaranteed for each $S_{i}$ :

1) There is a direct path (refer to Fig.5) for the vehicle through all the points in $S_{i}$, or,

2) There is at least one pair of adjacent points $(u, v)$ in $S_{i}$ where the length of any feasible Dubins path for the GDPP from $u$ to $v$ is at least equal to $\pi \rho$ where $\rho$ is the minimum turning radius of the vehicle.

Therefore, using this approximation algorithm, one can obtain a lower bound for the GDPP in the following way: Without loss of generality, suppose $S_{1}^{*}, S_{2}^{*}, \cdots, S_{l}^{*}$ denote the subsequences where the distances between any two adjacent points in the subsequences is at most equal to $2 \rho$ and there is at least one adjacent pair of points in each subsequence where the property 2 above is satisfied. The following is a then a lower bound for the GDPP:

$$
\sum_{i=1}^{n-1} d\left(t_{i}, t_{i+1}\right)+\sum_{i=1}^{l} \min _{(u, v) \in S_{i}^{*}}(\pi \rho-d(u, v))
$$

Algorithm: Relax-3-point

1) Divide the heading angles at alternate points into set of intervals $\mathcal{I}_{i}=\left\{I_{i 1}, \ldots I_{i p}\right\}$ for $i=1,3, \ldots(n-$ $1) / n$, as shown in Figure 4(a). If the number of points are odd, then the heading angles at the last point $t_{n}$ are discretized; if they are even, the angles at point $t_{n-1}$ are discretized.

2) Construct a graph $G^{2}$ as follows: add a node to $G^{2}$ corresponding to each interval $I_{i k} \in \mathcal{I}_{i}, i=$ $1,3 \ldots, k=1 \ldots p$; add an edge between any two nodes corresponding to interval $I_{i m}, I_{i+2 n}$, if they belong to successive interval sets $\mathcal{I}_{i}$ and $\mathcal{I}_{i+2}$; set the weight of the edge to be the distance of the Dubins path between the interval $I_{i m}$ to $I_{i+2 n}$ and that passes through the point $t_{i+1}$, which is computed using the procedure presented in Section III-B.

3) Add an auxiliary node $v_{0}$, and add edges connecting $v_{0}$ to all the nodes corresponding to the intervals $I_{1 k} \in \mathcal{I}_{1}$. Set the weight of all these edges to be zero.

4) If the number of points are odd, add an auxiliary node $v_{n+1}$, and add edges connecting all the nodes corresponding to the intervals in $\mathcal{I}_{n}$ to $v_{n+1}$; set the weight of these edges to be zero.

If the number of points are even, then add a node $\left(v_{n}\right)$ corresponding to the point $t_{n}$; add edges from each node corresponding to all the intervals in $\mathcal{I}_{n-1}$ to $v_{n}$. Set the weight of these edges to be the length of the Dubins paths from the corresponding interval $I_{(n-1) k} \in \mathcal{I}_{n-1}$ to the point $t_{n}$.

5) Compute the shortest path on $G^{2}$ from $v_{0}$ to $v_{n}$ (or $\left.v_{n+1}\right)$ using the Dijkstras algorithm.

where $d(u, v)$ denotes the Euclidean distance between any adjacent target points $u$ and $v$.

\section{Computational Results}

The computational experiments are run for two different problem sizes, instances with 11 and 21 target points. We have generated 25 instances for each case, the coordinates of the points are generated from an uniform distribution in an area of $1000 \times 1000$ units. The turning radius of the vehicle is chosen to be 100 units. The sequence of the points is chosen by solving a Hamiltonian path problem on the generated points. Here, we present the results for lower bound computation done using the two relaxation algorithms Relax-2-point and Relax-3-point presented in Seciton IV. Along with these, we compute the lower bounds using the result from [16], and the trivial Euclidean lower bound, which is the sum of the straight line distances. All the algorithms are coded in MATLAB and the computations are run on Dell Precision Workstation (Intel Xeon Processor 


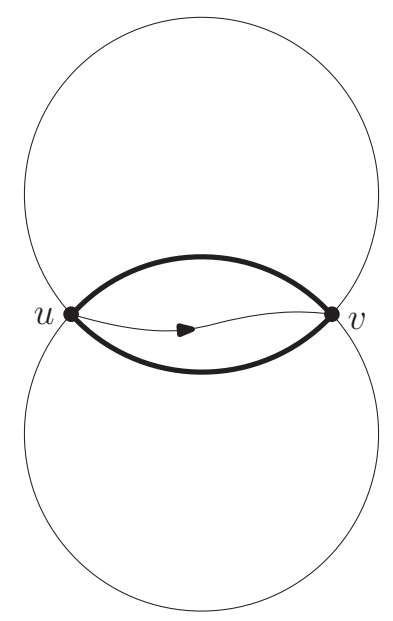

Fig. 5. A direct path between two points $u$ and $v$ that are at most $2 \rho$ apart is a path that lies in the shaded region connecting the two target points. Here, the radius of the circles that pass through the target points is equal to $\rho$. A feasible path $P$ through a sequence of points is referred to as a direct path if the part of $P$ that lies between any two adjacent points in $P$ is a direct path.

\section{@ $2.53 \mathrm{GHz}, 12 \mathrm{~GB}$ RAM).}

Figure 6 compares the lower bounds computed using the two algorithms Relax-3-point and Relax-2-point. The lower bound computed using the result from [16] and the trivial Euclidean lower bounds are shown. Clearly, relaxing at alternate points is fewer relaxations compared to the relaxing at every point. Fewer relaxations should produce a tighter bound by the Relax-3-point algorithm; this is corroborated by the lower bounds here. The lower bounds computed using the two algorithms presented are tighter than the bounds computed using Lee et. al's result. Along with the lower bounds, the upper bounds for the instances are shown in this figure. The upper bounds are computed using the following method

Upper bound computation: At each point, a discrete set $\Phi_{d}$ of headings are chosen by uniformly sampling from the set $[0,2 \pi],\left|\Phi_{d}\right|=128$. A graph is constructed (similar to the graph in the algorithm Relax-2-point) where each heading at each point corresponds to a node; nodes corresponding to successive target points are connected with edges with weight equal to the Dubins distance between those points with the corresponding headings. Two auxiliary nodes $\left(v_{0}, v_{n+1}\right)$ are added to the graph, node $v_{0}$ is connected to each of the nodes that corresponds to the first target point with zero cost edges. Node $v_{n+1}$ is connected to all the nodes correspond to the last target point with zero cost edges. The shortest path on this graph from $v_{0}$ to $v_{n+1}$ gives a feasible path for the GDPP. The length of this path is an upper bound to the optimal solution to the GDPP.

Figure 7 shows the lower bounds computed using the algorithm Relax-3-point and the trivial lower bound (Euclidean length) for the 25 instances. It shows the bounds computed using three different sets of discretization, 4,8 and 16 intervals respectively at each relaxed point. One can observe that as the discretization level increases, the lower bounds increases for every instance. It indicates that, as the intervals at target points are narrowed down to smaller size, the algorithm produces tighter lower bounds, and will eventually converge to the optimal solution of the problem.

The paths generated using the solution to the relaxed GDPP for one of the instances with 21 targets is shown in Figure 8 . The feasible path (upper bound) for this instance is shown using dashed lines and curves. At each relaxed point, the headings are divided into 8 uniform intervals, i.e. each interval size is $\pi / 4$. One can observe that the heading angles are discontinuous at some of the points in the relaxed path. But the difference between the incoming and outgoing headings is less than $\pi / 4$ at every point.

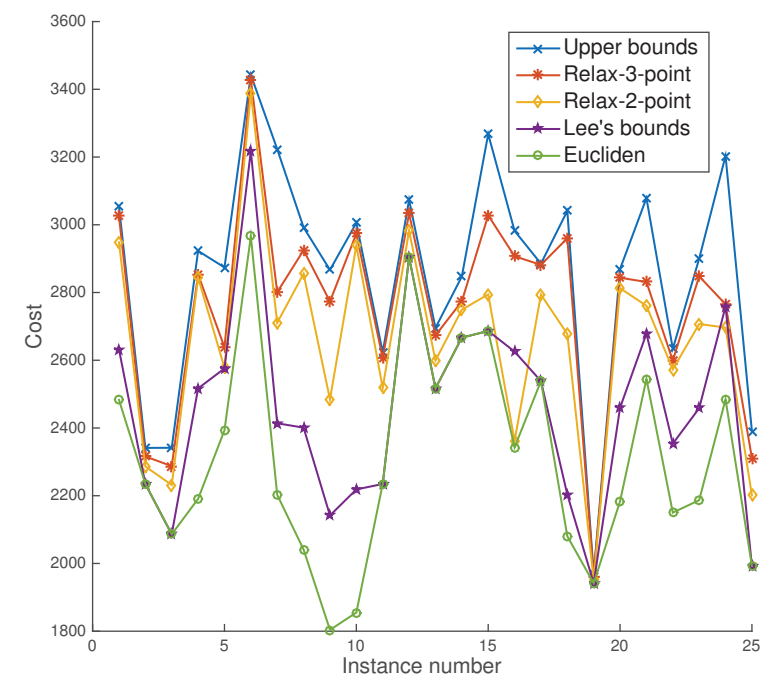

(a) Instances with 11 target points

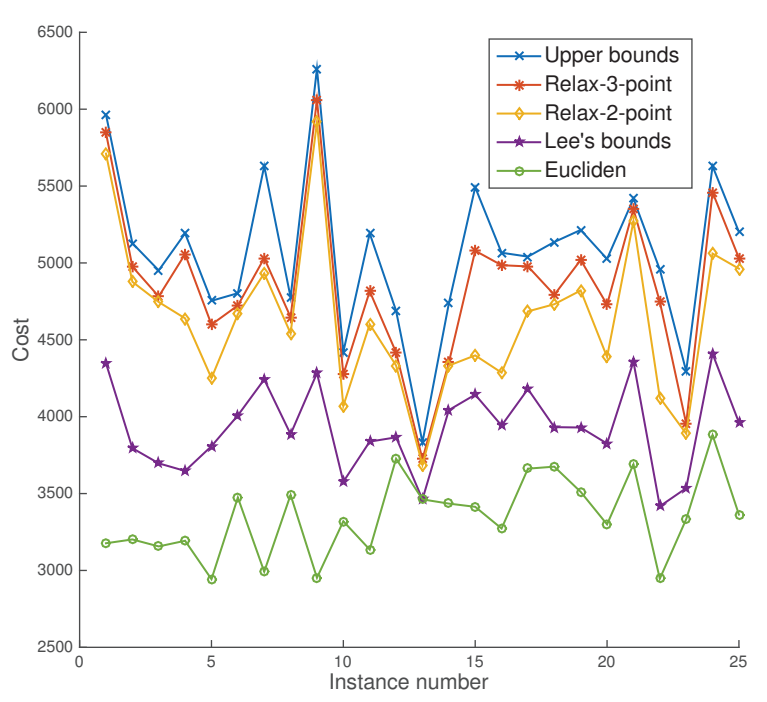

(b) Instances with 21 target points

Fig. 6. Comparison of the lower bounds computed using the two algorithms. The plots include the upper bounds and trivial lower bounds (Euclidean length). 


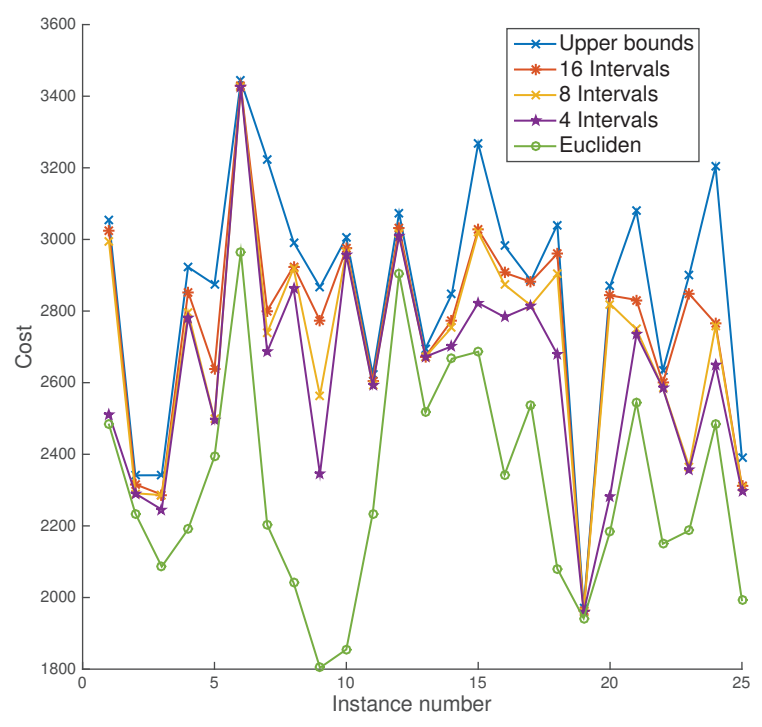

(a) Instances with 11 target points

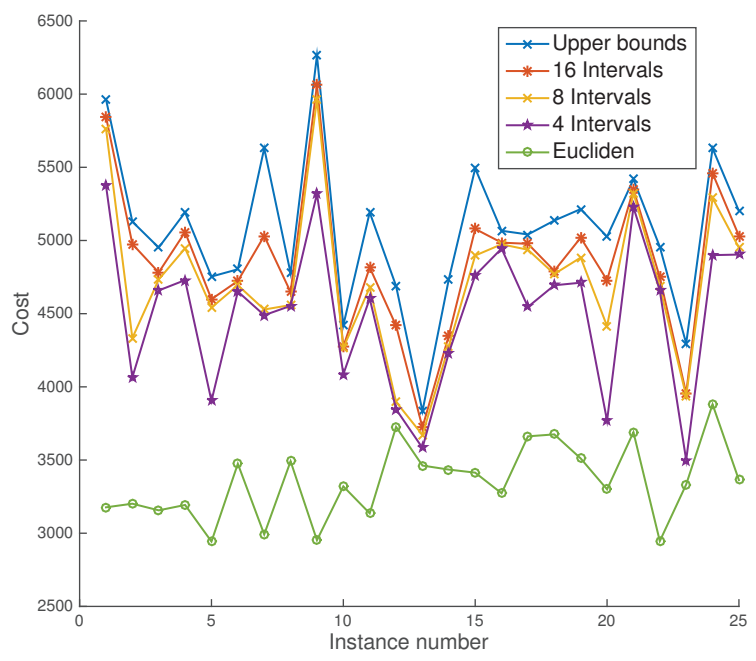

(b) Instances with 21 target points

Fig. 7. Lower bounds computed using interval discretization at alternate points (Relax-3-point) with 4,8 , and 16 intervals at each relaxed point

\section{CONCLUSIONS}

We presented algorithms to compute lower bounds to the problem of shortest path of bounded curvature passing through a sequence of points. We presented two different relaxations; the optimal solutions of these relaxed problems are lower bounds to the original shortest path problem. We presented the methods to solve the sub-problem that arise, where we need to find Dubins paths that starts at a point with heading restricted to an interval, passes through an intermediate point, and ends at a final point with the final heading constrained to be with in a specific interval. We solve this by discretizing the heading at the intermediate point, and solving separately the two Dubins paths. We presented

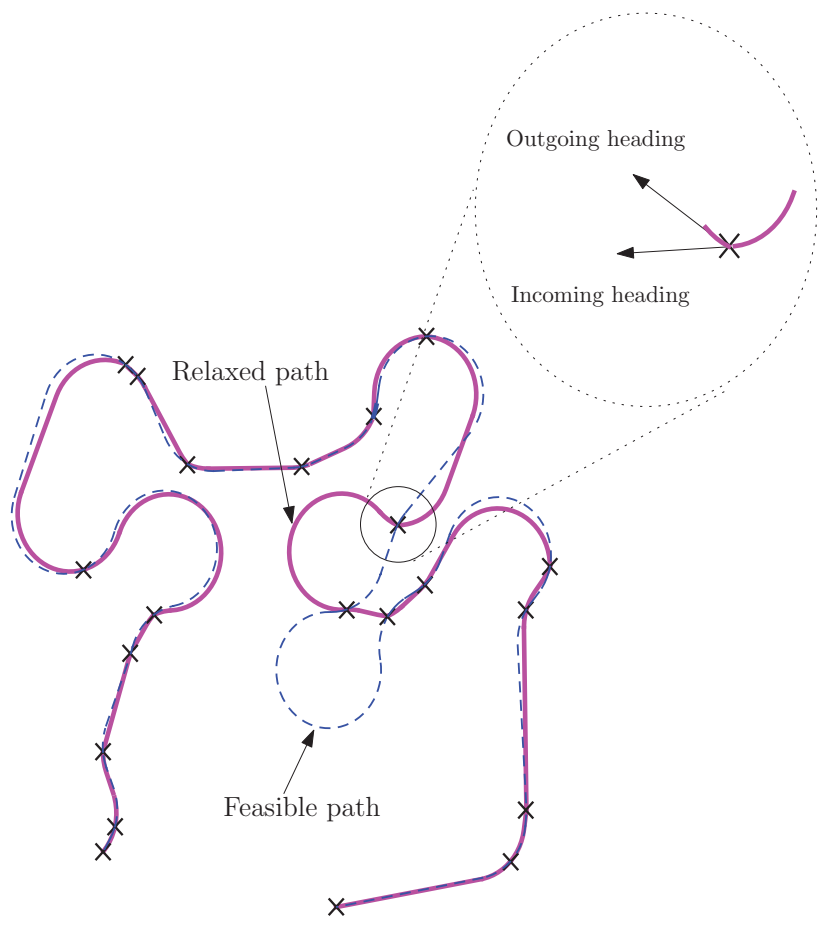

Fig. 8. Feasible Dubins path and the path from solving the relaxed GDPP

the computational results and compare the lower bounds computed with bounds from an existing result. These lower bounds are significantly tighter, and they are with in $10 \%$ of the best known upper bounds for every instance. A scope of improvement of this result lies in characterizing the Dubins paths that passes through three points using optimal control theory. This may lead to solving the sub-problem with less computational effort.

\section{REFERENCES}

[1] A. C. Medeiros and S. Urrutia, "Discrete optimization methods to determine trajectories for dubins' vehicles," Electronic Notes in Discrete Mathematics, vol. 36, pp. 17 - 24, 2010, \{ISCO 2010 - International Symposium on Combinatorial Optimization. [Online]. Available: http://www.sciencedirect.com/science/article/pii/ S1571065310000041

[2] D. Macharet and M. Campos, "An orientation assignment heuristic to the dubins traveling salesman problem," in Advances in Artificial Intelligence - IBERAMIA 2014, ser. Lecture Notes in Computer Science, A. L. Bazzan and K. Pichara, Eds. Springer International Publishing, 2014, vol. 8864, pp. 457-468.

[3] D. G. Macharet, A. Alves Neto, V. F. da Camara Neto, and M. F. Campos, "Efficient target visiting path planning for multiple vehicles with bounded curvature," in IEEE International Conference on Intelligent Robots and Systems (IROS), 2013, pp. 3830-3836.

[4] D. G. Macharet, A. A. Neto, V. F. da Camara Neto, and M. F. Campos, "Data gathering tour optimization for dubins' vehicles," in Congress on Evolutionary Computation (CEC), 2012, pp. 1-8.

[5] P. Sujit, B. Hudzietz, and S. Saripalli, "Route planning for angle constrained terrain mapping using an unmanned aerial vehicle," Journal of Intelligent \& Robotic Systems, vol. 69, no. 1-4, pp. 273-283, 2013

[6] R. J. Kenefic, "Finding good dubins tours for uavs using particle swarm optimization," Journal of Aerospace Computing, Information, and Communication, vol. 5, no. 2, pp. 47-56, 2008.

[7] D. G. Macharet, A. A. Neto, V. F. da Camara Neto, and M. F. Campos, "Nonholonomic path planning optimization for dubins' vehicles," in International Conference on Robotics and Automation (ICRA). IEEE, 2011, pp. 4208-4213. 
[8] Z. Tang and U. Ozguner, "Motion planning for multitarget surveillance with mobile sensor agents," IEEE Transactions on Robotics, vol. 21, no. 5, pp. 898-908, Oct 2005.

[9] S. Rathinam, R. Sengupta, and S. Darbha, "A resource allocation algorithm for multivehicle systems with nonholonomic constraints," IEEE Transactions on Automation Science and Engineering, vol. 4, pp. 98-104, 2007.

[10] L.E.Dubins, "On curves of minimal length with a constraint on average curvature, and with prescribed initial and terminal positions and tangents," American Journal of Mathematics, vol. 79, no. 3, pp. 487-516, 1957.

[11] J.-D. Boissonnat, A. Cérézo, and J. Leblond, "Shortest paths of bounded curvature in the plane," Journal of Intelligent and Robotic Systems, vol. 11, no. 1-2, pp. 5-20, 1994. [Online]. Available: http://dx.doi.org/10.1007/BF01258291

[12] H. J. Sussmann and G. Tang, "Shortest paths for the reeds-shepp car: a worked out example of the use of geometric techniques in nonlinear optimal control," Rutgers Center for Systems and Control Technical Report, vol. 10, pp. 1-71, 1991.

[13] J.-D. Boissonnat and S. Lazard, "A polynomial-time algorithm for computing shortest paths of bounded curvature amidst moderate obstacles," International Journal of Computational Geometry \& Applications, vol. 13, no. 03, pp. 189-229, 2003.

[14] P. K. Agarwal, P. Raghavan, and H. Tamaki, "Motion planning for a steering-constrained robot through moderate obstacles," in Symposium on Theory of Computing. ACM, 1995, pp. 343-352.

[15] P. K. Agarwal and H. Wang, "Approximation algorithms for curvatureconstrained shortest paths," SIAM Journal on Computing, vol. 30, no. 6, pp. 1739-1772, 2001.

[16] J.-H. Lee, O. Cheong, W.-C. Kwon, S. Shin, and K.-Y. Chwa, "Approximation of curvature-constrained shortest paths through a sequence of points," in Algorithms - ESA 2000, ser. Lecture Notes in Computer Science, M. Paterson, Ed. Springer Berlin Heidelberg, 2000, vol. 1879, pp. 314-325.

[17] X. Goaoc, H.-S. Kim, and S. Lazard, "Bounded-curvature shortest paths through a sequence of points using convex optimization," SIAM Journal on Computing, vol. 42, no. 2, pp. 662-684, 2013.

[18] P. Oberlin, S. Rathinam, and S. Darbha, "Today's traveling salesman problem," Robotics \& Automation Magazine, vol. 17, no. 4, pp. 70-77, 2010.

[19] J. Le Ny, E. Feron, and E. Frazzoli, "On the dubins traveling salesman problem." IEEE Transactions on Automatic Control, vol. 57, no. 1, pp. 265-270, 2012.

[20] X. Ma, D. Castañón, et al., "Receding horizon planning for dubins traveling salesman problems," in Conference on Decision and Control. IEEE, 2006, pp. 5453-5458.

[21] C. Epstein, I. Cohen, and T. Shima, "On the discretized dubins traveling salesman problem," Technical Report, 2014.

[22] S. G. Manyam, S. Rathinam, and S. Darbha, "Computation of lower bounds for a multiple depot, multiple vehicle routing problem with motion constraints," Journal of Dynamic Systems, Measurement, and Control, vol. 137, no. 9, p. 094501, 2015.

[23] S. Manyam and S. Rathinam, "A tight lower bounding procedure for the dubins traveling salesman problem," Presented at the International Symposium on Mathematical Programming, 2015. [Online]. Available: http://arxiv.org/abs/1506.08752

[24] S. Manyam, S. Rathinam, D. Casbeer, and E. Garcia, "Shortest paths of bounded curvature for the dubins interval problem," arXiv preprint arXiv:1507.06980, 2015. 